\title{
Difusión del conocimiento Desafíos para la Universidad Politécnica Salesiana
}

Ivonne López ${ }^{1}$

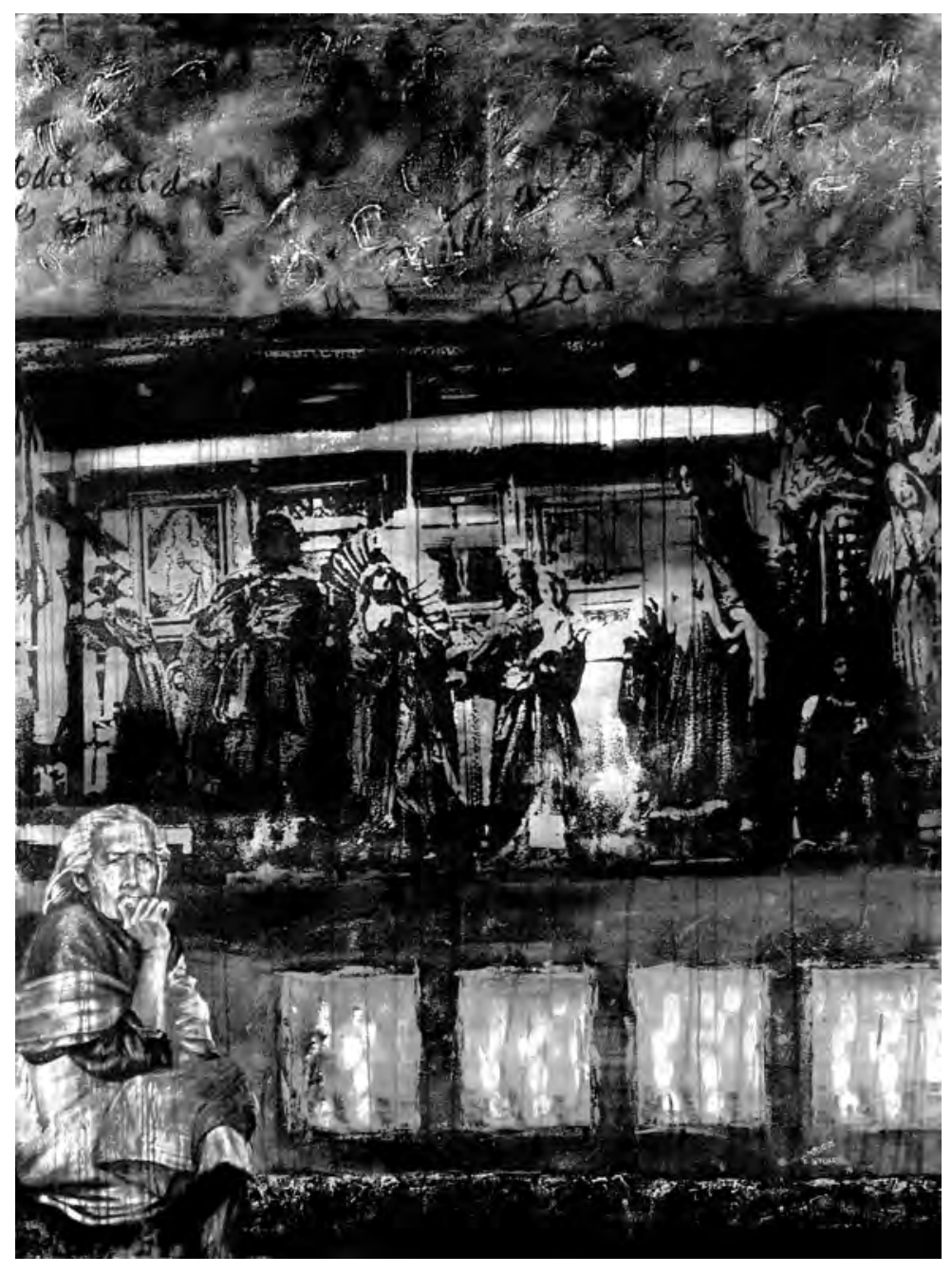

Rincón sin límite. Acrílico sobre tela. 1996

\footnotetext{
$\overline{1}$ Coordinación de diseño de material educativo para programas en modalidad virtual. UPS, Quito.
} 
Las estrategias y metodologías educativas que hacen uso de las tecnologías de la información y la comunicación en los distintos procesos de enseñanza-aprendizaje son el objeto de investigación actual. Quienes hacemos el equipo Educación Virtual en la Universidad Politécnica Salesiana, nos hemos sumado a estas líneas investigativas, así como al análisis y la discusión permanente respecto a la generación de dichos procesos, la sistematización y la difusión del conocimiento en el ámbito universitario, en el contexto ecuatoriano y latinoamericano.

La mayoría de los países de América Latina aportan débilmente al desarrollo de la Ciencia y la Tecnología, invierten en investigación menos del $0.5 \%$ del Producto Interno Bruto, es decir menos del $1 \%$ que recomienda la UNESCO y menos del 20\% de las universidades de estos países están en capacidad de realizar actividades de investigación científica (Erazo, 2007: 55).

Partimos de esta premisa para reflexionar sobre la Difusión del Conocimiento provocado por la universidad ecuatoriana en el ámbito general y por la Universidad Politécnica Salesiana en el ámbito local.

Por qué decir "provocado", porque creo que el término connota adecuadamente lo que debe ser el quehacer universitario en cuanto al conocimiento se refiere.

¿Qué es conocimiento? ¿Qué es ciencia? ¿Qué significa conocer? Y preguntando con más especificidad ¿qué es para la UPS el conocimiento y quiénes deben ser los detentadores del discurso científico?

\section{Aproximaciones históricas y epistémicas sobre el conocimiento}

A partir del surgimiento de la filosofía se plantea como avance el paso del conocimiento mítico al logos o conocimiento racional.

El ser humano llega a percibir que el universo y sus fenómenos están ordenados por leyes; y, el logos -la razón- permitirá el conoci- miento de la realidad de ese universo y de la "naturaleza de las cosas".

La razón entonces se instaura como principio divino que "crea, domina y dirige la naturaleza y el universo".

Desde entonces, también se determina la necesidad de formulaciones que otorguen veracidad a los conocimientos y por lo tanto, también estipulen la autoridad para elaborar y promulgar el discurso de la razón.

Las corrientes evolucionistas establecieron un sentido de progreso al desarrollo del pensamiento humano, al ubicar un primer estado teológico, luego un estado metafísico y por fin un estado científico del conocimiento.

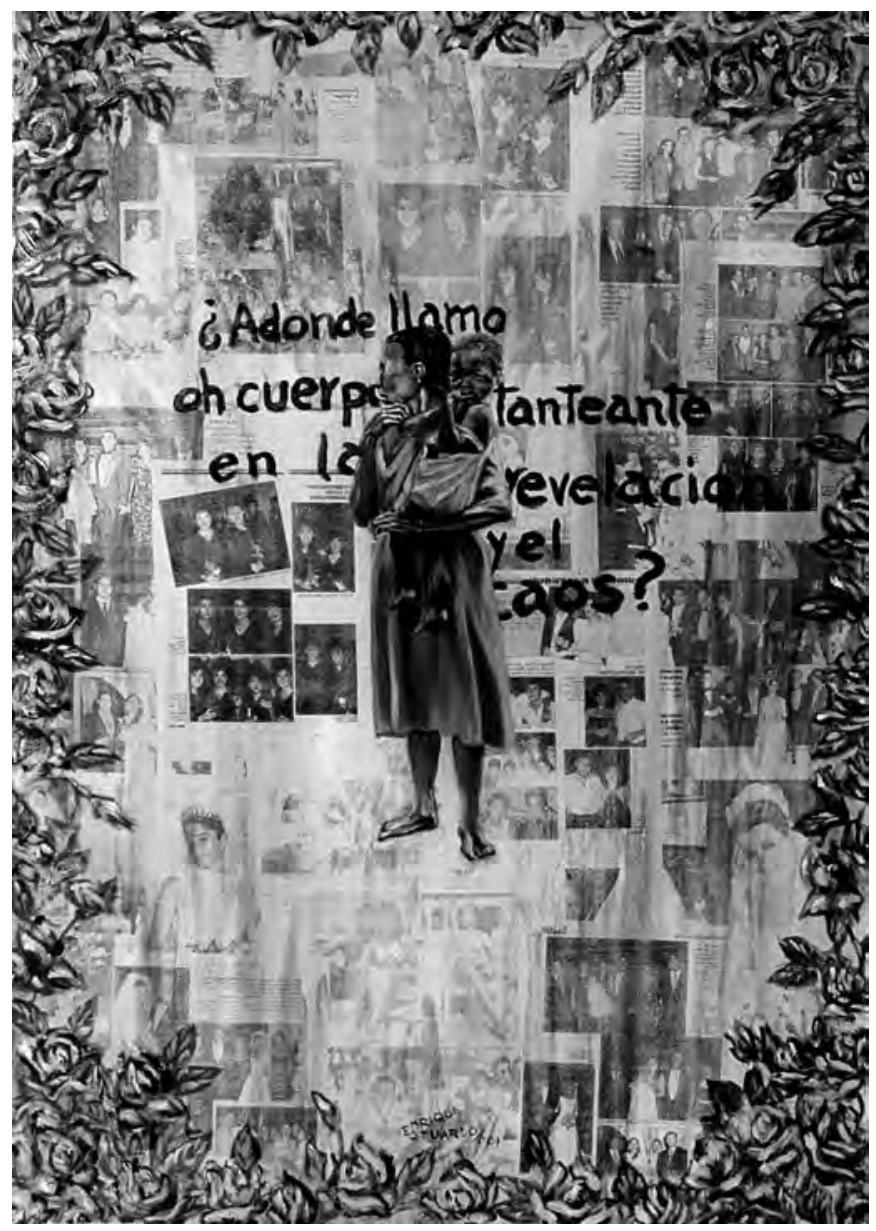

Vida rosa. Transferencia fotográfica y acrílico sobre tela. 2001 
El paradigma positivista determinó que:

- El acceso a la realidad se da a través de la percepción sensorial, la razón y del uso de instrumentos.

- El conocimiento válido es el que permite identificar causas y consecuencias evidenciadas en la realidad.

- El método científico y la repetición de la experiencia permiten establecer leyes y teorías de carácter universal.

Con el advenimiento de la revolución industrial, en el paso del siglo XVIII al XIX, los grandes sistemas de pensamiento articulados hasta entonces se ven obligados a un cambio de rumbo en la discusión sobre la prominencia de la razón respecto a la praxis.

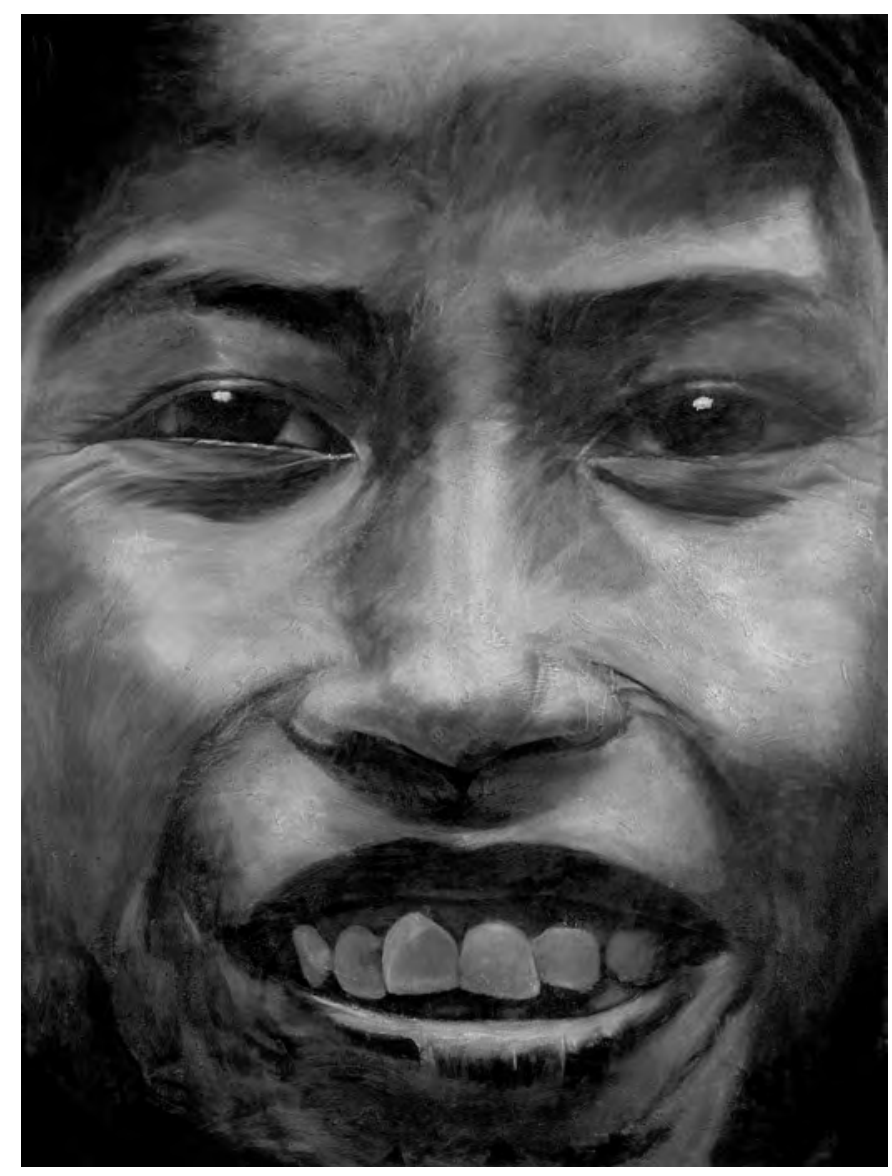

Quilago (32 módulos). Acrílico sobre tela. 2004
Hasta entonces, no se había evidenciado tanto que el conocimiento científico concebido como el conjunto de teorías, leyes, modelos, principios que permiten comprender, explicar y evidenciar la realidad, también podía ser aplicado para transformar drásticamente esa realidad.

La ciencia se pone al servicio de la innovación. La tecnología como aplicación de los conocimientos científicos se pone al servicio de la mecanización de los procesos productivos del naciente capitalismo industrial.

Ya conocemos el impacto que este fenómeno produjo en la organización social. Uno de ellos, es la necesidad de la incorporación de los sujetos a la educación formal. Sin embargo, se mantiene el mismo esquema bifurcado: la formación de unas personas dedicadas a la investigación científica y la aplicación tecnológica; y otras, a la adquisición de destrezas para asegurar los procesos productivos.

\section{Investigación científica y socie- dad de la información y el cono- cimiento}

En los últimos cien años, siglo XX y lo que va del siglo XXI la investigación científica ha ido cambiando el escenario tradicional para su desarrollo; ha pasado del espacio académico al espacio empresarial. De tal manera que, en el actual momento, el mayor impulso económico y de infraestructura para la actividad científica está en manos de grandes detentadores del poder político, económico y comercial.

Esta transición ha sido explicada como "Sociedad del Conocimiento". Esta noción aparece en la segunda mitad del siglo XX y tiene relación con el análisis de las sociedades posindustriales. El sociólogo Peter Drucker ya para los años noventa caracteriza una sociedad en la que el conocimiento constituye la fuente más importante de la productividad, el crecimiento económico y también, de las desigualdades sociales. 
Asimismo, se ha designado a esta época como la transición de una economía basada en los productos a una basada en los servicios, Bell (1973).

En este contexto, las tecnologías empiezan a tener una importancia mayúscula porque se convirtieron en la parte más visible de la ciencia.

De entre todas, las tecnologías de la información y la comunicación (TIC) han alcanzado protagonismo en varios espacios de la organización social, constituyéndose en aplicaciones designadas, sobre todo, a la transmisión y al consumo de la información.

Se consolidan así los medios masivos bajo el control de sistemas de dominio de circulación de discursos, incluidos los científicos, que asegurarán el mantenimiento del poder político, económico y social.

Las TIC se desarrollan como herramientas, recursos, entornos y servicios que permiten optimizar el manejo de la información y el desarrollo de la comunicación, a través de la retroalimentación, los modelos materiales y modelos virtuales, así como la estandarización de procesos y el enfoque sistémico.

La Internet, si bien en su origen cumplía con fines militares, se constituye ahora en un medio de integración de recursos escritos y audiovisuales, y en un espacio de circulación de la información en todo nivel. Además que ha posibilitado también la conformación de escenarios de articulación social, rebasando los límites de las distancias geográficas y las fronteras geográficas.

Esta articulación social también tiene distintos niveles de profundidad, en medida de los intereses y objetivos de personas, de grupos y comunidades. Ningún medio masivo hasta ahora había permitido los niveles de comunicación que este medio posibilita: recuperación del acceso a la información, libertad de circulación de discursos, participación, conformación de comunidades de aprendizaje y de militancia social.

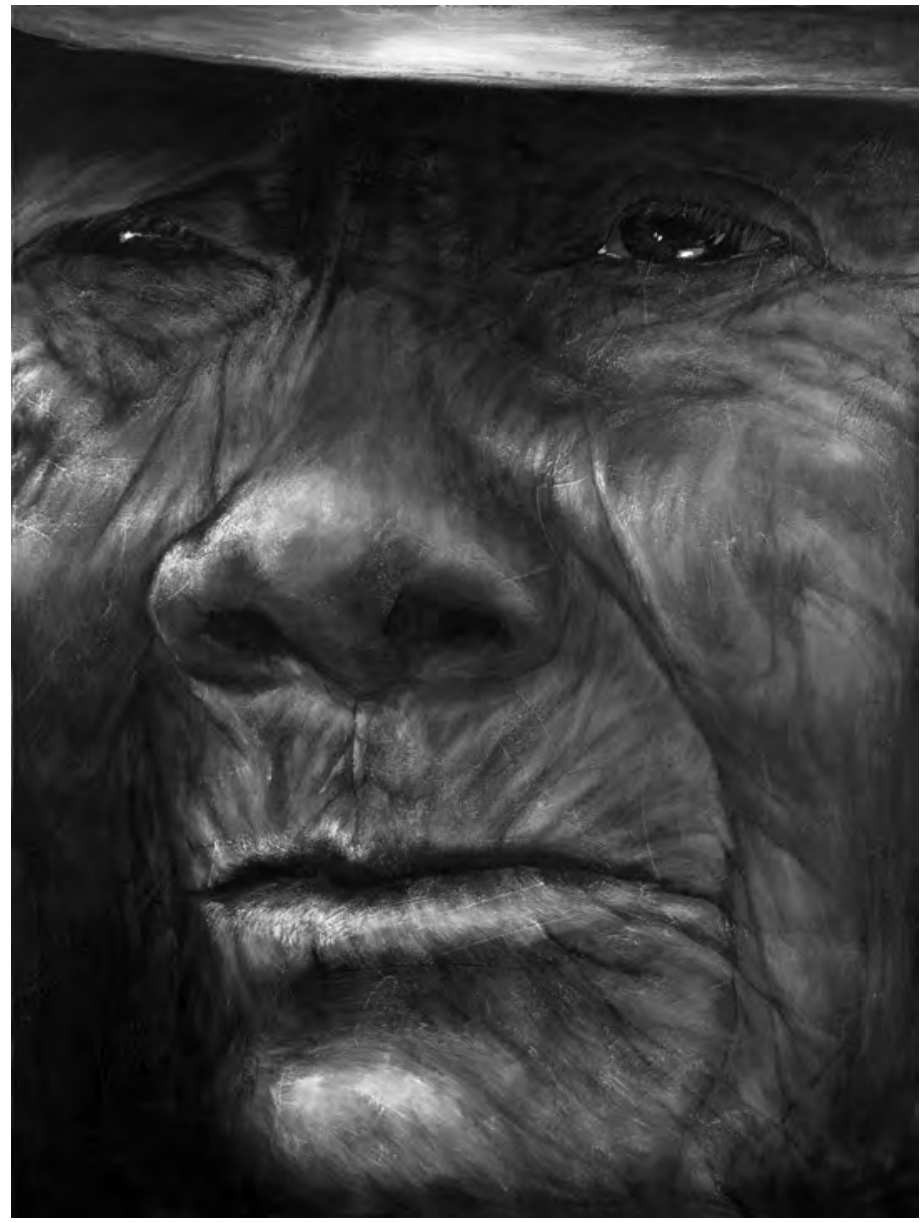

Quilago (32 módulos). Acrílico sobre tela. 2004

\section{La investigación científica en la educación superior}

Otro aspecto de análisis es que el desarrollo científico ha sido fragmentado y monodisciplinario. En este momento, se evidencia la necesidad de la coexistencia de las disciplinas aplicadas para el estudio de los mismos objetos y de la realidad.

Cifrando a La Rosa, conocimientos y metodologías divergentes se han ido acumulando, de manera que, nos asiste una demanda de una visión interdisciplinar y transdisciplinar para comprender holísticamente la realidad. 
Interdisciplinar, porque se reconoce la coexistencia y la interrelación de las distintas ciencias en el discurso científico.

Transdisciplinar, porque permite la construcción de un discurso integral sobre la realidad en el que cada disciplina aporte con justeza como propone Wolfendend (1999: 33) "una genuina integración entre las disciplinas enfocadas hacia una sinergia”.

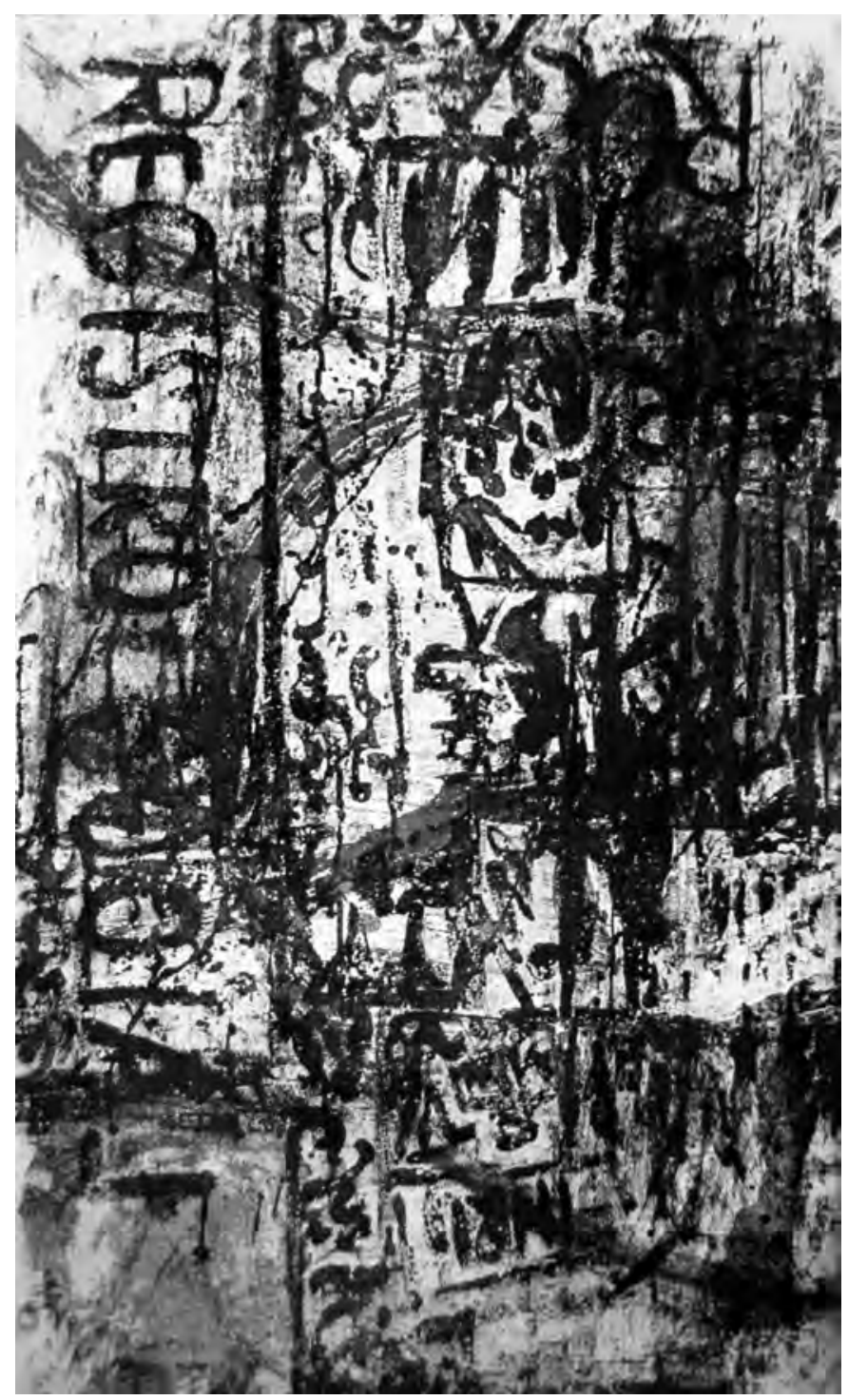

Escondidas voces. Técnica mixta. 1996
Respondo aquí la pregunta inicial ¿qué es el conocimiento para la Universidad Politécnica Salesiana y quiénes deben ser los detentadores del discurso científico?

El conocimiento científico es un sistema de leyes, teorías y principios que construidos socialmente permiten comprender y explicar holísticamente la realidad, de tal forma que podamos demostrarla y transformarla, mediante la experienciación y la aplicación en un contexto determinado. Por lo tanto, todos aquellos espacios donde se produzcan experiencias investigativas y se teorice sobre la realidad son un lugar para el discurso científico.

En la actualidad, la Universidad se ve abocada a tomar decisiones del tipo: ¿Qué actores sociales debemos formar? ¿Debemos hacer academia o debemos solamente profesionalizar? ¿Podremos liderar la creación y consolidación de una comunidad científica? ¿Se trata de contribuir a la construcción o a la homogeneización del conocimiento?

Si revisamos aquí los cuestionamientos que la realidad presenta a la Universidad podemos responder: en primer lugar que es necesario recuperar el liderazgo del medio académico en la investigación científica; como segunda premisa, que la Universidad es la entidad llamada a provocar espacios para el anhelado "diálogo entre saberes" que permita llegar a conocimientos que se articulen en una ciencia coherente y consecuente con el ser humano, así también, que sea correspondiente a los diversos contextos, y, en tercer lugar, que la Universidad debe establecer sus estrategias para la interacción social, la difusión del conocimiento y su aplicación en tecnologías igualmente consecuentes con la humanidad.

Para cumplir con estos objetivos, la universidad requiere hacer posible la constitución de una comunidad científica en la que los sujetos de la acción educativa puedan dar a conocer su discurso e incidir en la dinámica institucional interna; de manera que, la entidad educativa pueda ingresar a la discusión científica de alto 
nivel nacional e internacional y con mayor posibilidad de hacer impacto en la sociedad y concretar su propuesta de transformación de la realidad; una comunidad científica abierta a la permanente vinculación con los sectores sociales y gubernamentales.

Reconocer la importancia de la formación profesional pero no únicamente sustentada en los itinerarios y las competencias extraídos desde la demanda laboral sino que su fortaleza sea la rigurosidad científica y metodológica que permita la proposición de nuevas estrategias y modos de producción definitivamente humanistas. Es decir, ciudadanos que sean capaces de dar respuestas a realidades que cambian rápidamente.

Desarrollar procesos educativos centrados en el inter-aprendizaje, con rigurosidad académica y científica; con la aplicación adecuada, planificada y en la medida justa de las tecnologías; la participación activa de estudiantes y docentes en posibilidad de investigar, disentir y consensuar.

\section{Educación virtual: concreciones pedagógicas y tecnológicas}

La educación virtual es una de las propuestas que la Universidad Politécnica Salesiana ha venido gestando en los últimos años, a través de un trabajo inter y transdisciplinar de gestión académica y administrativa. Las acciones han sido planificadas en coherencia con los ejes estratégicos de la UPS: docencia, investigación, vinculación con la colectividad y gestión. Se ha desarrollado como una modalidad de estudios No presenciales mediados por entornos virtuales de aprendizaje, cuya metodología está fundamentada en cuatro ejes: pedagógico, comunicacional, cultural y tecnológico.

Pedagógico, en cuanto aplicamos en el diseño microcurricular el constructivismo social (Vigotsky), que propone la construcción de conocimiento a través de la interrelación entre los sujetos-interaprendizaje, la experiencia educati- va relacionada con el contexto y los conocimientos previos de los beneficiarios para provocar aprendizajes significativos, así como estrategias colaborativas de aprendizaje, donde todos los participantes contribuyen a la investigación, el análisis y la construcción del conocimiento.

Comunicacionales, en cuanto los actores se interrelacionan durante todo el proceso de enseñanza-aprendizaje, mediante estrategias de comunicación asincrónica y sincrónica permitiendo la flexibilización en los horarios de acceso a los espacios de encuentro; en esta propuesta, tanto docentes como estudiantes desarrollan

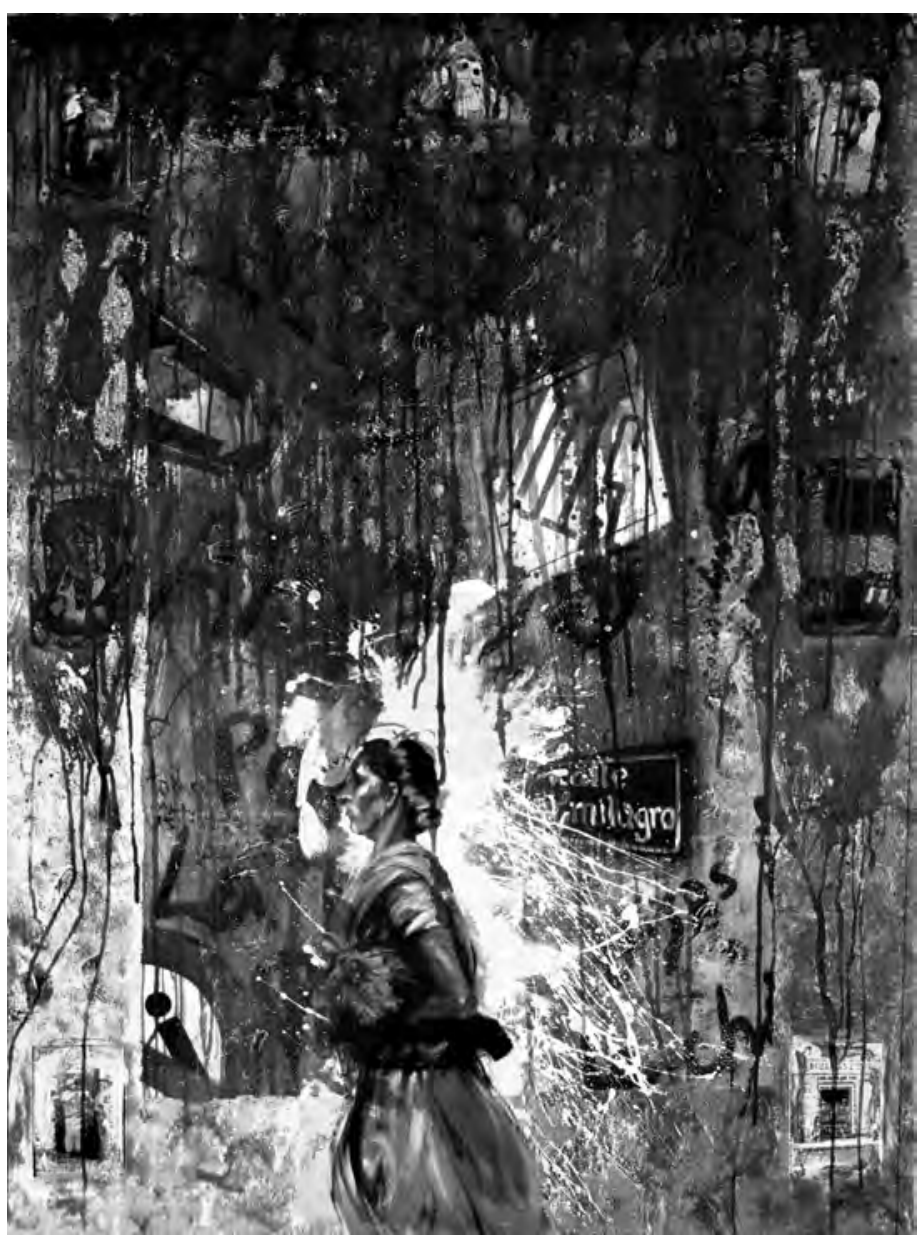

Proceso de olvido I. Acrílico sobre tela. 1996 
mayor destreza en la comunicación escrita. Pensamos que la educación sólo es posible en cuanto exista comunicación.

Cultural, en absoluto respeto al contexto, a los conocimientos locales y entendiendo al conocimiento como una construcción social que permite dar respuesta a las problemáticas del entorno.

Tecnológicos, en medida que cada una de las aplicaciones es analizada e implementada en correspondencia con las actividades y requerimientos que la planificación microcurricular exige. En consecuencia, la tecnología implementada debe favorecer la consecución de los fines pedagógicos y comunicacionales, para lo cual se ha optado por la utilización de Moodle, un software libre que consiste en un entorno virtual donde se integran espacios para la comunicación asincrónica como foros y mensajería interna y para comunicación sincrónica como el chat, igualmente, espacio de entrega de tareas y presentación de contenidos.

Por lo dicho anteriormente, no se puede considerar a la educación virtual una derivación de la educación a distancia cuyas estrategias están apoyadas fundamentalmente por materiales de estudio impresos o multimedios, la relación interpersonal es mínima a través de tutorías presenciales o por medio del uso del correo electrónico.

En la UPS el énfasis deberá estar en un relacionamiento horizontal donde el docente no es el único portador de la verdad; que los docentes participen de manera más creativa y propositiva, se dinamice el proceso diversificando las acciones, no se agoten en entrega de tareas y resolución de cuestionarios.

La propuesta del equipo de trabajo de educación virtual es que, precisamente, la labor de difusión del conocimiento en la Universidad, empieza con la acción de garantizar la "significatividad" de cada uno de los momentos del aprendizaje, de manera que la planificación racional y responsable es indispensable, se debe potenciar la investigación docente a través de la publicación de los contenidos desarrollados para cada asignatura y que son producto de su propia experiencia investigativa. Entendemos, que cada una de las personas que confían en nuestra propuesta educativa es precisamente un potencial promovedor de lo que en la UPS se logra.

\section{Difusión del conocimiento: decisiones y acciones institucionales}

A partir de los hechos, se abren algunas posibilidades para la difusión del conocimiento dentro y fuera de la U PS.

En principio la construcción de modelos que integren políticas, programas, métodos y estrategias para la comunicación de la ciencia y la tecnología, tanto en el mismo ámbito académico, como hacia la sociedad en general.

Porque, las comprensiones de la realidad del mundo y del ser humano que se dan en cada experiencia así como las aplicaciones propuestas respecto a estas elaboraciones, deben ser sometidas a la crítica y la evaluación social para hacer efectivo el derecho a la opinión sobre el tipo de ciencia y las aplicaciones que tienen prioridad para resolver los problemas de la humanidad. Nos sumamos, a lo que dice Ignacio Fernández Bayo citado por Erazo (2007): "La ciencia debe ser objeto de crítica, control y denuncia".

Sugerimos entonces, definir políticas para la gestión del conocimiento que incluyan:

- Criterios para la identificación y caracterización de los conocimientos que se desarrollan en la Universidad Politécnica Salesiana.

- Capitalizar esos conocimientos que se dan tanto en los equipos de investigación conformados por docentes, como aquellos que se producen en las aulas. Es decir, docentes y estudiantes autores de contenidos científicos.

- Trabajar en estrategias y metodologías para que la comunicación de los conocimientos científicos llegue a quienes no de- 
tentan el lenguaje especializado sin perder calidad ni precisión.

- Diversificar los canales de difusión, sin agotar las acciones a través de medios masivos y tradicionales como la publicación impresa.

- Aprovechar las posibilidades que da la Internet para llegar con nuestro aporte al mundo entero.

- Liderar una red de instituciones universitarias en Latinoamérica que acuerden la liberación de contenidos científicos producidos en cada entidad; para que se visibilice la investigación y se someta a la crítica académica, con la finalidad de elevar el nivel de rigurosidad científica.

- Fortalecer, actualizar permanente y difundir la existencia de colecciones bibliográficas, documentales, impresas y audiovisuales en nuestra institución académica con el fin de que sean un referente a nivel latinoamericano.

- Valorar y promover el "capital intelectual" institucional.

- Priorizar el uso de software libre como estrategia inicial para enfrentar la dependencia tecnológica.

- Asumir la formación de docentes con capacidad de discernir la información científica válida disponible en los distintos medios sobre todo en la Internet.

- Promover la conformación de una comunidad científica dentro de la comunidad uni- versitaria sostenida en principios de pluralidad, respeto entre colegas, transparencia en las acciones, responsabilidad social.

- Crear bibliotecas y repositorios virtuales con contenidos completos.

- Promover que docentes y estudiantes construyan blogs, para la socialización y construcción continua del conocimiento.

\section{Bibliografía}

- ERAZO, María de los Ángeles: Comunicación, divulgación y periodismo de la ciencia. Quito: Editorial Planeta. 2007

\section{Bibliografía de Internet}

- KRÜGER, Karsten. Daniel Bell citado en El advenimiento de la sociedad post-industrial. En: Concepto de Sociedad del Conocimiento. http://www.ub.es/geocrit/b3w-683.htm. Consulta: 2009-05-15. Universidad de Barcelona.

Filosofía Griega. En http://www.e-torredebabel.com/Historia-de-la-filosofia/Filosofiagriega/Platon/Ciencia.htm. Consulta: mayo 2009.

- LA ROSA, Amparo. Una perspectiva transdisciplinaria para analizar la comunicación en la era digital. Lima, s/f. En: http://www.razonypalabra.org.mx/anteriores/n48/bienal/mesa10.pdf. Consulta: mayo 2009. 